\title{
Tratar ou extrair? Tratamento de lesão endoperiodontal, um relato de caso clínico
} Treat or extract? Endoperiodontal lesion treatment, a case report

¿Tratar o extraer? Tratamiento de la lesión endoperiodontal, reporte de un caso

\section{Gabriela Sumie Yaguinuma GONÇALVES ${ }^{1}$}

Tayna Natsumi TAKAKURA ${ }^{1}$

Anderson CATELAN ${ }^{2}$

Rosalinda Tanuri Zaninotto VENTURIM ${ }^{3}$

Carolina dos Santos SANTINONI ${ }^{3}$

Christine Men MARTINS ${ }^{3}$

${ }^{1}$ Curso de Graduação em Odontologia, Universidade do Oeste Paulista (UNOESTE). Campus Presidente Prudente, 19050-920 Presidente Prudente - SP, Brasil

${ }^{2}$ Professor do Curso de Graduação em Odontologia e do Programa de Pós-Graduação em Odontologia - Clínica Odontológica, Universidade do Oeste Paulista (UNOESTE). Campus Presidente Prudente, 19050-920 Presidente Prudente - SP, Brasil

${ }^{3}$ Professora do Curso de Graduação em Odontologia, Universidade do Oeste Paulista (UNOESTE). Campus Presidente Prudente, 19050-920 Presidente Prudente - SP, Brasil

\section{Resumo}

Introdução: Lesões endoperiodontais são lesões originadas de produtos inflamatórios encontrados tanto em periodonto quanto em polpa. Tais lesões podem se originar devido a uma infecção pulpar ou periodontal. Visando o prognóstico favorável, é imprescindível o conhecimento da etiologia, realização do correto diagnóstico e elaboração do plano de tratamento que envolve o tratamento endodôntico precedido do tratamento periodontal. Objetivo: O propósito do presente trabalho foi de relatar um caso clínico de lesão endoperiodontal e o tratamento realizado. Relato de caso clínico: Paciente gênero feminino, 51 anos, compareceu à clínica com uma fístula na região do dente 46, procedeuse com exame radiográfico, rastreamento de fístula, testes endodônticos e avaliação periodontal. Foi diagnosticada lesão endoperiodontal. Executou-se, então, o tratamento endodôntico em sessões múltiplas, utilizando hidróxido de cálcio como medicação intracanal e o tratamento periodontal concomitante; finalizou-se endodontia obturando-se os canais radiculares. Conclusão: Observou-se, no controle, que a associação de tratamentos foi eficaz e houve melhora significativa do quadro, constatando-se silêncio clínico e sucesso do tratamento. Realizar o tratamento conservador a despeito da exodontia foi a melhor escolha para a paciente.

Descritores: Endodontia; Periodontia; Polpa Dentária; Periodonto.

\section{Abstract}

Introduction: Endoperiodontal lesions are lesions originated from inflammatory products found in both periodontal and pulp tissue. Such lesions may originate due to a pulp or periodontal infection. Aiming at a favorable prognosis, it is essential to know the etiology, perform a correct diagnosis and elaborate the treatment plan that involves endodontic treatment preceded by periodontal treatment. Objective: The purpose of the present study was to report a clinical case of endoperiodontal injury, and the treatment performed. Case report: Female patient, 51 years old, attended the clinic with a fistula in the region of tooth 46. It was performed a radiographic examination, fistula screening, endodontic tests and periodontal evaluation. Endoperiodontal lesion was diagnosed. Endodontic treatment was performed in multiple sessions, using calcium hydroxide as intracanal medication and concomitant periodontal treatment; endodontic treatment was completed by filling the root canals. Conclusion: At the control appointment, it was observed that the combination of treatments was effective and there was a significant improvement of the condition, noting clinical silence and treatment success. Doing a conservative treatment despite extraction was the best choice for the patient.

Descriptores: Endodontics; Periodontics; Dental Pulp; Periodontium.

\section{Resumen}

Introducción: Las lesiones endoperiodontales son lesiones originadas por productos inflamatorios que se encuentran tanto en la periodoncia como en la pulpa. Dichas lesiones pueden originarse debido a pulpa o periodontal infección. Con el objetivo de un pronóstico favorable, es esencial conocer la etiología, hacer el diagnóstico correcto y elaborar el plan de tratamiento que implica el tratamiento endodóntico precedido por el tratamiento periodontal. Objetivo: El propósito del presente estudio fue informar un caso clínico de lesión endoperiodontal y el tratamiento realizado. Informe del caso: Una paciente de 51 años llegó a la clínica con una fístula en la región del diente 46. Fue realizado examen radiográfico, examen de fístula, pruebas de endodoncia y evaluación periodontal. Se diagnosticó lesión endoperiodontal. El tratamiento endodóntico se realizó en múltiples sesiones, utilizando hidróxido de calcio como medicación intracanal y tratamiento periodontal concomitante; La endodoncia se completó obturando los conductos radiculares. Conclusión: En el control, se observó que la combinación de tratamientos fue efectiva y hubo una mejora significativa de la condición, con silencio clínico y éxito del tratamiento. La realización de un tratamiento conservador a pesar de la extracción fue la mejor opción para el paciente.

Descriptores: Endodoncia; Periodoncia; Pulpa Dental; Periodoncio.

\section{INTRODUÇÃO}

Os problemas de natureza endodôntica ou periodontais têm sido tratados como especialidades separadas ${ }^{1}$, no entanto, muitas vezes, podem ocorrer concomitantemente e, nesse momento, profissionais das duas especialidades devem se unir e tratar a doença a fim de manter o dente no arco $^{2}$.

Existe uma íntima relação entre endodontia e periodontia, sendo que um pode interferir no outro ${ }^{3}$. O deslocamento de produtos metabólicos da polpa comprometida para o periodonto dá-se por meio do forame apical, canais laterais, secundários ou canal interradicular. E o inverso é verdadeiro, visto que uma inflamação/infecção do ligamento periodontal pode comprometer a polpa por meio do forame, sendo essa segunda condição menos prevalente ${ }^{1}$.

Para o comprometimento apical por meio de um problema endodôntico, inicialmente deve-se haver uma infecção cariosa que leva ao comprometimento pulpar. As bactérias presentes nessa infecção, bem como seus produtos e subprodutos, adentram no sistema de canais radiculares, alcançando os níveis mais apicais. Nesse momento, pode ocorrer uma inflamação periapical e, na persistência desse quadro infeccioso/inflamatório, evoluir para uma lesão periapical que compromete os tecidos de sustentação dentários ${ }^{3,4}$.

Por sua vez, um estado simples de gengivite, se persistente, pode evoluir para a destruição dos tecidos de suporte periodontais, também atingindo os níveis apicais. Nesse momento, há uma infecção por microrganismos periodontopatogênicos do forame periapical que danifica a estrutura pulpar, levando à 
sua inflamação e subsequente necrose $\mathrm{e}^{3,4}$.

Em ambas situações, nos estados evoluídos, há a presença de uma lesão periodontal e endodôntica combinada. No entanto, durante sua evolução, pode ainda não haver o comprometimento dos dois tecidos. Dessa forma, o diagnóstico vem a ser a ferramenta principal para delimitar o plano de tratamento correto a fim de se conseguir alcançar o sucesso ${ }^{3,4}$.

Para se fazer o diagnóstico diferencial entre as duas patologias, alguns aspectos devem ser observados $^{5}$. Sinais de sensibilidade pulpar são importantes para a detecção da origem do problema, portanto, diversos são os testes que podem ser realizados a fim de se observar a presença ou ausência de sensibilidade pulpar. Frente à ausência, pode-se considerar que há o comprometimento da polpa. Nesse mesmo sentido, a observância de cáries, restaurações extensas ou insatisfatórias e presença de radiolucência periapical podem também indicar alterações patológicas endodônticas. A avaliação periodontal vem acrescentar dados importantes. Profundidade de bolsas periodontais assim como suas localizações associadas à presença de cálculos e biofilme bacteriano pode indicar alterações patológicas periodontais. A somatória desses indícios tende a nos levar ao diagnóstico correto ${ }^{3-5}$.

Por meio dessas avaliações, pode-se classificar as lesões endoperiodontais em 5 tipos: 1) Lesão endodôntica primária; 2) Lesão endodôntica primária com envolvimento periodontal secundário; 3) Lesão periodontal primária; 4) Lesão periodontal primária com envolvimento endodôntico secundário; 5) Lesão endoperiodontal verdadeira ${ }^{6}$.

$\mathrm{Na}$ lesão endodôntica primária, a polpa encontra-se necrosada. A drenagem faz um trajeto fistuloso que passa pelo sulco gengival através de uma tumefação na gengiva inserida. Por meio dessa fístula é possível inserir o cone de guta percha para rastrear a origem do problema. Frequentemente, a destruição óssea e comprometimento do suporte é mínimo, restringindo-se apenas à fistula. Como a lesão primária é um problema endodôntico que se manifestou no ligamento periodontal, o tratamento restringe-se apenas ao tratamento radical da polpa, sem nenhuma associação com o tratamento periodontal $^{3-6}$.

A lesão periodontal primária é caracterizada pela presença de vitalidade pulpar. A lesão periodontal está presente, evoluindo em direção apical, levando a formação de defeitos ósseos e subsequente aspecto radiográfico de lesão periapical, além da presença de mobilidade dentária, perda de inserção generalizada, acúmulo de placa e cálculo. $\mathrm{O}$ tratamento restringe-se apenas à terapia periodontal, sem tratamento endodôntico associado. $\mathrm{O}$ prognóstico de sucesso depende do comprometimento do periodonto de inserção e da capacidade do paciente de responder ao tratamento ${ }^{3-6}$.
No entanto, há a evolução de ambas as doenças supracitadas, pode haver o envolvimento conjunto da doença periodontal com a doença endodôntica. Nesses casos, a resolução do caso dá-se por meio do tratamento de ambas as condições, sendo que inicialmente deve-se realizar a neutralização do conteúdo tóxico/necrótico do canal radicular e da colocação de uma medicação intracanal, seguido da raspagem supra e subgengival, tratamento periodontal completo e da obturação definitiva do sistema de canais. O prognóstico de sucesso dependerá do comprometimento ósseo e das estruturas de suporte ${ }^{3-6}$.

O presente caso clínico tem como objetivo relatar um caso clínico de lesão endoperiodontal e o tratamento realizado, o qual teve sucesso.

\section{CASO CLÍNICO}

Paciente oriental, gênero feminino, 51 anos de idade, compareceu à clínica da Faculdade de Odontologia do Oeste Paulista (FOOP) para tratamento odontológico; sua queixa principal incluía apenas a insatisfação perante a estética de seus dentes. No entanto, após a propedêutica clínica (anamnese e exame físico intrabucal e extrabucal), detectou-se uma fístula na região do dente 46 e, após questionada, a paciente relatou que não se recordava de ter apresentado qualquer quadro de sintomatologia dolorosa nessa área, porém, há cerca de uma semana, a região do terço inferior do lado direito de sua face esteve edemaciado; notou-se, com o decorrer dos atendimentos, que a paciente em questão apresentava alto limiar de dor. Procedeu-se com exame radiográfico (Figura 1A), rastreamento de fístula (Figura 1B), testes endodônticos e avaliação periodontal. Durante o rastreamento de fístula com cone de guta percha, foi constatado que o trajeto fistuloso caminhava em direção ao meio do dente, próximo à região de furca. $\mathrm{O}$ dente em questão também apresentava uma restauração de resina composta extensa e insatisfatória nas faces mésioocluso-distal (Classe II) e vestibular (Classe V), sendo que essa última estava acarretando em um quadro inflamatório com consequente sangramento gengival devido à desadaptação ao término cervical, impossibilitando uma adequada sondagem da área; pelo motivo expresso, a restauração de resina composta da face vestibular foi removida e substituída provisoriamente por cimento de ionômero de vidro anteriormente ao tratamento, a fim de se obter uma adequação do meio bucal e regredir a inflamação. Os testes endodônticos (percussão vertical, percussão horizontal e palpação) responderam positivamente e o teste térmico (frio), negativamente. Durante a avaliação periodontal, verificou-se perda de inserção nos dentes anteriores inferiores, sequela de uma antiga doença periodontal localizada já não mais ativa. Foi diagnosticada, 
portanto, lesão endoperiodontal combinada verdadeira. Executou-se, então, o tratamento endodôntico em sessões múltiplas, utilizando hidróxido de cálcio como medicação intracanal (Figura 1 C) e o tratamento periodontal concomitante por meio de raspagem e alisamento radicular supragenvival e subgengival mediante irrigação com tetraciclina. O preparo mecânico foi realizado com a lima Reciproc r25 e seu respectivo motor, sabendo que o comprimento real de trabalho (CRT) do dente 46 era de $17 \mathrm{~mm}$ para todos os canais (MV, ML e D); o preparo químico deu-se com o uso de hipoclorito de sódio $2,5 \%$. Foi realizada a PUI, ou seja, a agitação do hipoclorito de sódio 2,5\% e o EDTA com o instrumento Easyclean a fim de se remover a smear layer e expor os túbulos dentinários à medicação de escolha, que foi o hidróxido de cálcio. Enquanto a ação da medicação intracanal estava sendo conseguida, foi realizada a raspagem e alisamento corono-radicular do dente em questão, com o uso da tetraciclina concomitante. Após 30 dias, finalizou-se, então, a endodontia obturando-se os canais radiculares por meio da técnica Híbrida de Tagger (Figura 1D).
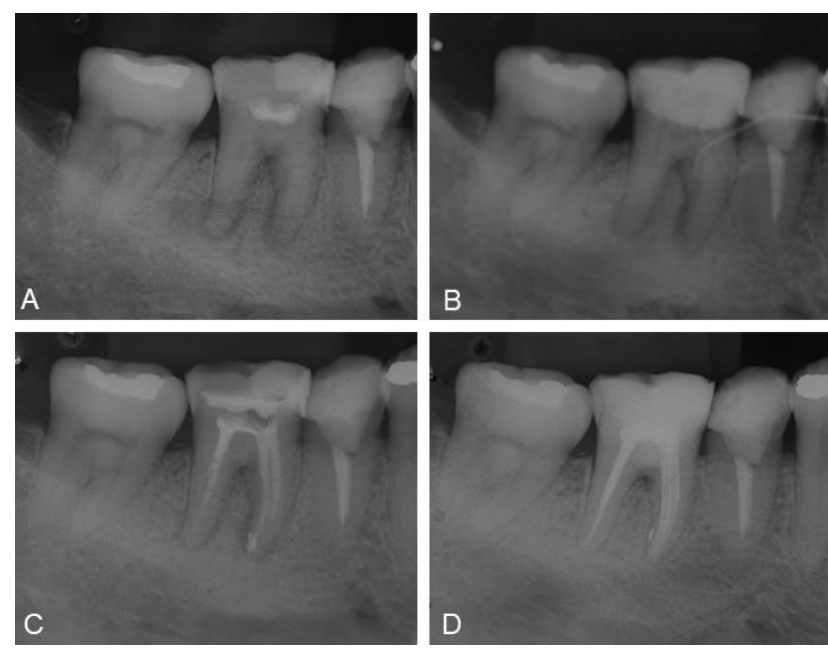

Figura 1: Caso clínico de lesão endoperiodontal. (A) Caso clínico inicial. (B) Rastreamento da fístula. (C) Medicação intracanal a base de hidróxido de cálcio. (D) Obturação e proservação de 6 meses.

Findados seis meses do tratamento, foi realizada a proservação e observou-se que a associação de tratamentos foi eficaz e houve melhora significativa do quadro, constatando-se silêncio clínico e sucesso do tratamento. A restauração foi realizada provisoriamente na face oclusal com resina Bulk Fill Flow; avaliações e considerações estão sendo estudadas para dar continuidade na reabilitação do paciente e entre as opções de tratamento, as melhores indicações são a prótese parcial fixa (pino de fibra de vidro + coroa) ou de onlay.

DISCUSSÃO

As lesões periodontais-endodônticas representam um desafio diagnóstico e terapêutico complexo para o cirurgião dentista. Para se chegar a um diagnóstico adequado da lesão endoperiodontal, faz-se necessário realizar um exame clínico cuidadoso, incluindo histórico completo do paciente, exame clínico abrangente e uso de radiografias dentárias. Apesar de ser imperativo fornecer a terapia adequada na sequência correta do tratamento, nem sempre é possível fazer um diagnóstico preciso; em alguns casos, a terapia endodôntica ou periodontal sozinha pode ser suficiente, no entanto, em outros casos, uma combinação de terapia endodôntica e periodontal pode ser necessária para tratar com sucesso o caso ${ }^{7}$.

Lesões endoperiodontais podem ser tratadas por cuidados endodônticos e periodontais e, às vezes, complementada por cirurgia, a solução terapêutica dependerá do grau de comprometimento das estruturas dentárias ${ }^{8,9}$. Recomenda-se um tempo adequado para a cicatrização do tecido antes da reavaliação para não haja precipitação ao se realizar a terapia adicional ${ }^{10}$.

O tratamento de lesões periodontaisendodônticas através da realização do tratamento do canal radicular antes do tratamento periodontal mostrou-se eficaz no caso descrito, indo à favor do que mostram diversos estudos, resultando em melhorias nos parâmetros clínicos, juntamente com ganhos ósseos alveolares ${ }^{11}$.

Segundo Estrela ${ }^{12}$, os critérios clínicos e radiográficos empregados pela Associação Americana de Endodontia para a análise do sucesso endodôntico destacam: ausência de dor e edema, ausência de sintomas à percussão e palpação, mobilidade fisiológica normal, ausência de drenagem e fechamento de fístula, dente em função e com fisiologia normal, desaparecimento de rarefação óssea periapical e estrutura óssea periapical normal, presença de selamento coronário perfeito, ausência de periodontite apical e silêncio clínico. Assim, após as etapas dos procedimentos endodônticos, estes aspectos foram observados.

$\mathrm{O}$ preparo químico-mecânico (PQM) caracteriza-se pela associação da instrumentação e irrigação do sistema de canais radiculares que, por sua vez, é capaz de reduzir, significativamente, a microbiota. No entanto, alguns microrganismos podem persistir e/ou permanecerem viáveis em áreas inacessíveis ao PQM, como em ramificações, istmos e túbulos dentinários. Assim, em muitos casos, faz-se necessário o uso de medicação intracanal entre sessões, a fim de favorecer a redução de microroganismos e suas toxinas, promovendo aumento significativo do sucesso no tratamento ${ }^{3,13}$.

O hidróxido de cálcio é o medicamento mais usado como medicação intracanal na endodontia $a^{3,14,15}$ por possuir várias características adequadas ${ }^{16}$ : é biocompatível $^{14,17}$, tem capacidade de elevação do $\mathrm{pH}$ do meio, colaborando com a alcanilização das áreas de reabsorção ${ }^{16}$ e de auxiliar na indução da formação de tecido mineralizado ${ }^{13,14}$, atua sobre vários 
microrganismos, inativando a endotoxina liberada pelas bactérias gram negativas, ou seja, possui ação antimicrobiana $^{14,18-20}$, tem propriedade de difusão no sistema de canais radiculares ${ }^{14,21}$ e promove o reparo apical e periapical ${ }^{21-23}$.

O medicamento inserido dentro do canal, em contraposição à substância química empregada na irrigação, terá tempo de ação intracanal suficiente para agir em profundidade e ter potencial para redução da microbiota em áreas não afetadas pelo $\mathrm{PQM}$, aspectos que podem colaborar com a reparação dos tecidos ${ }^{3}$.

Portanto, optou-se por sessões múltiplas visando somar ao preparo químico-mecânico as ações anti-inflamatórias e antimicrobianas da medicação intracanal, indução de reparo por tecido mineralizado e atuação como barreira física no interior dos canais radiculares do hidróxido de cálcio; além de que, durante a primeira sessão, verificou-se que o canal distal apresentava-se único no terço coronal, sofria uma bifurcação no terço médio e unia-se novamente no terço apical, tornando sua instrumentação mais complexa.

Nas lesões combinadas, a sequência clássica sugerida por Bergenholtz e Hasselgren ${ }^{24}$ é: 1) tratamento endodôntico dos canais radiculares; 2) tratamento básico periodontal: raspagem e polimento supragengival; 3) período de observação, através do qual a extensão da cura da área do periodonto possa ser determinada. Essa sequência visa, inicialmente, uma melhora das condições periodontais, permitindo adequada cicatrização e evitando a remoção de tecido viável durante instrumentação periodontal, e uma orientação para as medidas de higienização dos dentes, para o posterior tratamento periodontal subgengival. Estudos de Harrington et al. ${ }^{25}$, também preconizam e recomendam o tratamento da lesão endodôntica seguida da terapia periodontal, compreendendo o acesso cirúrgico e preparo do canal radicular, colocação de medicação intracanal (hidróxido de cálcio) nas alterações periódicas, orientações de higiene oral ao paciente, controle periódico, verificação dos resultados clínicos convenientes (redução da profundidade à sondagem e acréscimo da inserção clínica), terapia periodontal básica (raspagem supra e subgengival) podendo-se fazer uso de irrigação subgengival com antimicrobianos como adjunto à terapia periodontal não-cirúrgica, como o cloridrato de tetraciclina, afim de auxiliar na sanificação de áreas de difícil acesso26 e obturação do canal radicular, conforme realizado no presente relato de caso; o tratamento endodôntico deve ocorrer antes da terapia periodontal pois proporciona tempo para a cicatrização periapical inicial, possibilitando uma melhor avaliação das condições periodontais nos casos de lesões combinadas verdadeiras $^{27}$.

Existem outras terapias que estão sendo propostas na literatura que podem ser empregadas, dependendo da gravidade de cada caso, como alternativa à terapia periodontal básica, visando o tratamento desse tipo de lesão, contribuindo para o aumento das taxas de sucesso ${ }^{28}$, como a regeneração tecidual guiada ${ }^{29-31}$, utilizando, por exemplo, fibrina rica em plaquetas leucocitárias ${ }^{2}$, o manejo microcirúrgico endodôntico e periodontal, o enxerto ósseo e as técnicas de barreira de membrana ${ }^{32,33}$, além da irradiação com laser de $\operatorname{diodo}^{34}$, o manejo do defeito de furca com vidro bioativo ${ }^{35}$ e enxerto de tecido conjuntivo subepitelial com periósteo ${ }^{36}$. Sendo que, atualmente, também estão sendo utilizados kits de detecção de bactérias produtoras de peptidase para verificar a diminuição de bactérias na furca e canais radiculares ${ }^{37}$.

O prognóstico das lesões endo-periodontais dependerá do diagnóstico, tratamento e cronicidade da lesão, bem como da duração do envolvimento periodontal e consequente comprometimento ósseo e das estruturas de suporte ${ }^{7}$. Concomitante com a adesão do paciente, de modo que este realize uma excelente higiene bucal, pratique os cuidados necessários para manter a saúde e aceite e compreenda o tratamento.

Em geral, quando a doença primária de um tecido, isto é, polpa ou periodonto, está presente e a doença secundária está apenas começando é recomendado tratar a doença primária. A terapia endodôntica precede a terapia periodontal, sendo que a última pode ou não ser necessária, dependendo do estado da doença. A estratégia de tratamento deve focar na infecção pulpar e a realizar desbridamento e desinfecção do sistema de canais radiculares; a segunda fase inclui um período de observação, em que a extensão da cura periodontal resultante do tratamento endodôntico é seguida. Profundidade de sondagem reduzida pode normalmente ser esperada dentro de algumas semanas, enquanto a regeneração óssea pode exigir vários meses antes de ser detectada radiograficamente. Assim, a terapia periodontal, com e sem cirurgia periodontal, deve ser adiada até que o resultado do tratamento endodôntico possa ser adequadamente avaliado ${ }^{38}$.

Os autores responsáveis pelo tratamento da paciente, bem como a mesma, mostraram-se satisfeitos com o resultado favorável do caso apresentado e convencidos de que a escolha de uma abordagem conservadora e minimamente invasiva foi a mais apropriada. Manter um dente na boca significa, além de proporcionar estética, recuperar função; tem efeito no psicológico do indivíduo que, por sua vez, recupera a saúde de seu órgão e o mantem na cavidade bucal, propiciando o retorno do paciente ao convívio social, sem medo ou discriminação, melhorando, por conseguinte, sua qualidade de vida ${ }^{39}$. 


\section{CONCLUSÃO}

O correto diagnóstico provou-se o passo prioritário e mais importante para o sucesso na terapia odontológica em casos de lesão endoperiodontal, além da adesão e cooperação do paciente, associados à decisão terapêutica mais adequada para cada caso. A identificação precisa dos fatores etiológicos durante o exame clínico e radiográfico, além da necessidade da interação das duas especialidades, endodontia e periodontia, para se alcançar um prognóstico favorável, que, por sua vez, dependerá do diagnóstico, tratamento e cronicidade da lesão, bem como da duração do envolvimento periodontal e consequente comprometimento ósseo e das estruturas de suporte. O tratamento de lesões periodontais-endodônticas por meio da realização do tratamento do canal radicular antes do tratamento periodontal mostrou-se eficaz, resultando em melhorias nos parâmetros clínicos, juntamente com ganhos ósseos alveolares.

\section{REFERÊNCIAS}

1. Sunitha VR, Emmadi $P$, Namasivayam A, Thyegarajan R, Rajaraman V. The periodontal endodontic continuum A review. J Conserv Dent. 2008;11(2):54-62.

2. Betancourt P, Elgueta R, Fuentes R. Treatment of endo-periodontal lesion using leukocyte-plateletrich fibrin - a case report. Colomb Med. 2017;48(4):204-7.

3. Lopes HP, Siqueira JF. Endodontia: Biologia e Técnica. Rio de Janeiro: Medsi-Guanabara Koogan; 2015.

4. Lindhe J, Karring T, Lang NP. Tratado de periodontia clínica e implantologia oral. Rio de Janeiro: Guanabara Koogan; 2010.

5. Anand V, Govila V, Gulati M. Endo-perio lesion part II (the treatment) - a review. Archdent. 2012;3(1):10-6.

6. Rotstein I, Simon JH. Diagnosis, prognosis and decision-making in the treatment of combined periodontal-endodontic lesions. J Periodontol. 2004;34:165-203.

7. Parolia A, Gait TC, Porto ICCM, Mala K. Endoperio lesion: a dilemma from 19th until 21st century. J Interdisp Dent. 2013;3(1):2-11.

8. Kim E, Song JS, Jung IY, Lee SJ, Kim S. Prospective clinical study evaluating endodontic microsurgery outcomes for cases with lesions of endodontic origin compared with cases with lesions of combined periodontal-endodontic origin. J Endod. 2008;34(5):546-51.

9. Heasman PA. An endodontic conundrum: the association between pulpal infection and periodontal disease. Br Dent J. 2014;216(6):275-9.

10. Schmidt JC, Walter C, Amato M, Weiger R. Treatment of periodontal-endodontic lesions--a systematic review. J Clin Periodontol. 2014; 41(8):779-90.
11. Jivoinovici R, Suciu I, Dimitriu B, Perlea P, Bartok R, Malita M, Ionescu C. Endo-periodontal lesion--endodontic approach. J Med Life. 2014;7(4):542-44.

12. Estrela C. Endodontia laboratorial e clínica, Série Abeno: Odontologia Essencial - Parte Clínica. São Paulo: Artes Médicas; 2013.

13. Vera J, Siqueira JF Jr, Ricucci D, Loghin S, Fernández N, Flores B et al. One-versus two-visit endodontic treatment of teeth with apical periodontitis: a histobacteriologic study. J Endod. 2012;38(8):1040-52.

14. Mohammadi Z, Dummer PMH. Properties and applications of calcium hydroxide in endodontics and dental traumatology. Inter Endod $\mathrm{J}$. 2011;44(8):697-730.

15. Batista VES, Olian DA, Mori GG. Diffusion of hydroxyl ions from calcium hydroxide and aloe vera pastes. Braz Dent J. 2014;25(3):212-16.

16. Pereira TC, da Silva Munhoz Vasconcelos LR, Graeff MSZ, Ribeiro MCM, Duarte MAH, de Andrade FB. Intratubular decontamination ability and physicochemical properties of calcium hydroxidepastes. Clin Oral Investig. 2019;23(3):1253-62.

17. Andolfatto C, da Silva GF, Cornélio AL, Guerreiro-Tanomaru JM, Tanomaru-Filho M, Faria G, Bonetti-Filho I, Cerri PS. Biocompatibility of intracanal medications based on calcium hydroxide. ISRN Dent. 2012;2012:904963.

18. Duque TM, Prado M, Herrera DR, Gomes BPFA. Periodontal and endodontic infectious/inflammatory profile in primary periodontal lesions with secondary endodontic involvement after a calcium hydroxide-based intracanal medication. Clin Oral Investig. 2019;23(1):53-63.

19. Kim D, Kim E. Antimicrobial effect of calcium hydroxide as an intracanal medicament in root canal treatment: a literature review - Part I. In vitro studies. Restor Dent Endod. 2014; 39(4):241-52.

20. Adl A, Motamedifar M, Shams MS, Mirzaie A. Clinical investigation of the effect of calcium hydroxide intracanal dressing on bacterial lipopolysaccharide reduction from infected root canals. Aust Endod J. 2015;41(1):12-6.

21. Hilton TJ, Ferracane JL, Mancl L; Northwest Practice-based Research Collaborative in Evidence-based Dentistry (NWP). Comparison of $\mathrm{CaOH}$ with MTA for direct pulp capping: a PBRN randomized clinical trial. J Dent Res. 2013;92(7 Suppl):16S-22S.

22. Labban N, Yassen GH, Windsor LJ, Platt JA. The direct cytotoxic effects of medicaments used in endodontic regeneration on human dental pulp cells. Dent Traumatol. 2014; 30(6):429-34. 
23. McIntyre PW, Wu JL, Kolte R, Zhang R, Gregory RL, Bruzzaniti A, Yassen GH. The antimicrobial properties, cytotoxicity, and differentiation potential of double antibiotic intracanal medicaments loaded into hydrogel system. Clin Oral Investig. 2019;23(3):1051-59.

24. Bergenholtz, G., Hasselgren, G. Endodontics and periodontics. In: Lindhe, K., Karring, T., Lang, N. Clinical periodontology and implant dentistry. Copenhagen:Munksgaard; 2015.

25. Harrington GW, Steiner DR, Ammons WF. The periodontal-endodontic controversy. Periodontol 2000. 2002;30:123-30.

26. Fernandes LA, Martins TM, Almeida JM, Nagata MJ, Theodoro LH, Garcia VG, Bosco AF. Experimental periodontal disease treatment by subgingival irrigation with tetracycline hydrochloride in rats. J Appl Oral Sci. 2010;18(6):635-40.

27. Storrer CM, Bordin GM, Pereira TT. How to diagnose and treat periodontal endodontic lesions? RSBO. 2012;9(4):427-33.

28. Verma PK, Srivastava R, Gupta KK, Srivastava A. Combined endodontic periodontal lesions: A clinical dilema. J Interdiscip Dent. 2011; 1(2):119-24.

29. Oh SL, Fouad AF, Park SH. Treatment strategy for guided tissue regeneration in combined endodontic-periodontal lesions: case report and review. J Endod. 2009;35(10):1331-36.

30. Malli R, Lele P, Vishakha. Guided tissue regeneration in communicating periodontal and endodontic lesions - a hope for the hopeless. J Indian Soc Periodontol. 2011;15(4):410-13.

31. Ghezzi C, Virzì M, Schupbach P, Broccaioli A, Simion M. Treatment of combined endodonticperiodontic lesions using guided tissue regeneration: clinical case and histology. Int $\mathbf{J}$ Periodontics Restorative Dent. 2012;32(4):433-9.

32. Sun J, Liu Q. [Bio-Oss collagen bone grafting in the treatment of endodontic-periodontic lesion]. Nan Fang Yi Ke Da Xue Xue Bao. 2009;29(9):1905-6.

33. Sharma R, Hegde V, Siddharth M, Hegde R, Manchanda G, Agarwal P. Endodonticperiodontal microsurgery for combined endodontic-periodontal lesions: An overview. J Conserv Dent. 2014;17(6):510-16.

34. Li Y, Wang X, Xu J, Zhou X, Xie K. [The clinical study on the use of diode laser irradiation in the treatment of periodontal-endodontic combined lesions]. Hua Xi Kou Qiang Yi Xue Za Zhi. 2012;30(2):161-64, 168.

35. Narang S, Narang A, Gupta R. A sequential approach in treatment of perio-endo lesion. $\mathrm{J}$ Indian Soc Periodontol. 2011;15(2):177-80.

36. Pereira AL, Orzechowski PR, Filho SB, Cortelli JR. Subepithelial connective tissue graft: an alternative application for treating endoperiodontal lesions. Gen Dent. 2013;61(2):50-3.

37. Yoneda M, Motooka N, Naito T, Maeda K, Hirofuji T. Resolution of furcation bone loss after non-surgical root canal treatment: application of a peptidase-detection kit for treatment of type I endoperiodontal lesion. J Oral Sci. 2005; 47(3):143-47.

38. Shenoy N, Shenoy A. Endo-perio lesions: diagnosis and clinical considerations. Indian $\mathbf{J}$ Dent Res. 2010;21(4):579-85.

39. Gerritsen AE, Allen PF, Witter DJ, Bronkhorst EM, Creugers NH. Tooth loss and oral healthrelated quality of life: a systematic review and meta-analysis. Health Qual Life Outcomes. 2010;8:126.

\section{CONFLITO DE INTERESSES}

Os autores declaram não haver conflitos de interesse.

\section{AUTOR PARA CORRESPONDÊNCIA}

\section{Christine Men Martins}

Curso de Graduação em Odontologia

Universidade do Oeste Paulista (UNOESTE). Campus Presidente Prudente, Rua José Bongiovani, 700 19067-175 Presidente Prudente - SP, Brasil Tel.: +55 (18) 3229 - 2003,

e-mail: christinemen@ hotmail.com

Submetido em 03/10/2019

Aceito em 20/04/2020 